Keywords: Borderline personality disorder; Childhood abuse; Emotional neglect.

\title{
Coprophagia in a patient with borderline personality disorder
}

\author{
Hilario Blasco-Fontecilla, $\mathrm{PhD}^{\mathrm{a}, \mathrm{e}}$ \\ Rebeca Garcia-Nieto, PsyD ${ }^{b, c}$ \\ Raquel Alvarez-Garcia, MD $^{\mathrm{b}}$ \\ Laura Mata-Iturralde, MD ${ }^{\mathrm{b}}$ \\ Paula Artieda-Urrutia, MDa \\ Carlos Blanco, PhD $^{d}$ \\ Enrique Baca-Garcia, $\mathrm{PhD}^{\mathrm{b}, \mathrm{d}, \mathrm{e}, *}$ \\ a Department of Psychiatry, IDIPHIM-Puerta \\ de Hierro University Hospital, Autonoma \\ University, Madrid \\ b Department of Psychiatry, IIS-Jimenez \\ Diaz Foundation, Autonoma University, \\ Madrid \\ ${ }^{c}$ Department of Psychiatry, New York \\ University Langone Medical Center \\ d Department of Psychiatry, New York State \\ Psychiatric Institute, New York, New York \\ e CIBERSAM, Madrid, Spain \\ SPAIN \\ USA
}

\begin{abstract}
Background and Objectives: Human coprophagia is a rare phenomenon with severe medical and social consequences. So far, coprophagia has mainly been associated with severe mental retardation, schizophrenia, dementia, and depression. We report a case of coprophagia in a 30-year-old woman with Borderline Personality Disorder (DSM-IV). This case report illustrates the severity of symptoms and maladaptive social consequences of severe personality disorders, comparable to those of patients with schizophrenia. Pharmacological interventions and, particularly intensive psychotherapy might be effective for patients diagnosed with borderline personality disorder displaying severe behavior disorders. The treatment of choice for coprophagia is aversive behavioral intervention.
\end{abstract}




\section{Scientific Letter}

Human coprophagia is a rare phenomenon. Usually associated with severe psychiatric disorders such as mental retardation, dementia or schizophrenia ${ }^{1,2}$, we present a case of coprophagia in a 30-year-old woman with Borderline Personality Disorder (DSMIV). This case report illustrates the severity of symptoms and maladaptive social consequences of severe personality disorders.

Ms. L was admitted because she was exhibiting aggressive and coprophagic behavior. At admission, Ms. L's complete blood count revealed a mild normocytic anemia (Hbg: $11.7 \mathrm{~g} / \mathrm{dl}, \mathrm{MCV}$ : 99.3). Iron studies were as follows: iron: $46 \mathrm{ug} / \mathrm{dl}$ (range: $35-180 \mathrm{ug} / \mathrm{dl}$ ); ferritin: $28 \mathrm{ng} / \mathrm{ml}$ (range: $15-150 \mathrm{ng} / \mathrm{ml}$ ); TIBC: $170 \mathrm{mg} / \mathrm{dl}$ (range: $280-380 \mathrm{mg} / \mathrm{dl}$ ). The patient also presented with low serum protein $(5.2 \mathrm{~g} / \mathrm{l})$, albumin $(3.0 \mathrm{~g} / \mathrm{l})$, and glucose $(73 \mathrm{mg} / \mathrm{dl})$. Serum folate, Vitamin B12, $\mathrm{TSH}$, parathormone and thyroid hormone were within normal range. She had negative serologies for hepatitis and HIV. CT scan and EEG were normal.

According to the patient, she began to eat her own feces and drink her urine "to save money". Both parents reported having witnessed their daughter eating her feces. During evaluation, Ms. L tended to minimize the situation, exhibiting belle indifference, a rather blasé attitude about her symptoms and their consequences. She did not overtly displayed coprophagia during hospitalization, but once defecated in a litter. General treatment included risperidone up to 9 milligrams a day and supportive psychotherapy.

Ms. L reported having felt abandoned in her childhood because his father traveled due to his job. Ms. L's academic performance was good. She endorsed binge eating and food restriction episodes in adolescence. At
25 , she met her current partner and started using cannabis, cocaine, and hallucinogens. To date, she has manifested a pervasive pattern of job and interpersonal instability.

Parents reported history of fire setting, truancy, frequent lying, stealing, and recurrent physical fights. Besides, Ms. L attempted suicide once by drinking bleach after an argument with her boyfriend. The patient's mother also has a history of one failed suicide attempt by hanging.

Mrs. L was administered the WAIS-III ${ }^{3}$. She obtained a normal performance. On the Mini International Neuropsychiatric Interview $^{4}$, she did not meet criteria for any psychiatric disorder. She was also administered the Millon Clinical Multiaxial Inventory-III. Of note, she endorsed the items specific to childhood abuse.

We decided to assess the patient using the Minnesota Multiphasic Personality Inventory2 (MMPI-2). Ms. L's MMPI-2 profile was within the normal range. However, she obtained pathological scores in most validity scales.

Given her defensive attitude, we opted for administering her some projective tests; specifically, we used the Rorschach and the Thematic Apperception Test (TAT). The responses given by the patient indicated that her emotions were usually shallow. She appeared to be more emotional than rational. With regard to the TAT, her responses suggested that fear of abandonment plays an important role in her mental life.

Human coprophagia is a rare phenomenon with severe medical and social consequences. So far, coprophagia has mainly been associated with severe mental retardation ${ }^{5}$, schizophrenia $^{1}$, dementia $^{2}$, and depression ${ }^{6}$. Medical disorders related to coprophagia include chronic infestation of intestinal parasites, cerebral tumors, and seizure disorders ${ }^{2}$. Co- 
prophagia has also been related to nutritional deficits $^{7}$. The laboratory findings (mild normocytic anemia; iron parameters within the low range of normal level) might have played a role in the development of coprophagia in our patient as a way of re-ingesting essential nutrients. Unfortunately, we lack analyses preceding the episodes of coprophagia. Furthermore, coprophagia might also be explained as a consequence of her disturbed personality profile.

The patient was diagnosed with a Borderline Personality Disorder (DSM-IV) at discharge. The most prominent traits were impulsivity, affective instability, difficulty controlling anger, and suicidal behavior. The patient also endorsed personality traits compatible with other personality disorders, mainly antisocial (i.e., truancy), and histrionic (i.e., belle indifférence). Indeed, comorbidity is the rule among inpatients with Borderline Personality Disorder ${ }^{8}$.

The case of Ms. L exemplifies that the burden of disease in patients diagnosed with a severe personality disorder may match that of individuals with schizophrenia. Personality disorders are associated with poor quality of life ${ }^{9}$. Pharmacological interventions and, particularly intensive psychotherapy has proved to be effective for them ${ }^{10}$. Unfortunately, the severity of personality disturbance and psychiatric symptoms displayed by Ms. L, and her poor interpersonal functioning are poor prognostic factors. In this context, coprophagia might be considered a marker of severity. The treatment of choice of coprophagia is aversive behavioral intervention ${ }^{2}$. Because coprophagia is such a rare event, there are many aspects of this disturbing behavior that remain obscure. The pathophysiology of coprophagia deserves further research.

\section{Acknowledgments}

We thank Rosa Maria de Almeida Nunes, research assistant, for assistance in manuscript preparation and editing.

\section{Competing interests}

Dr. Blasco-Fontecilla has received lecture fees from Eli Lilly, AB-Biotics, and Shire. Dr. Baca-García, Ms. Garcia-Nieto, and Mrs. Alvarez-Garcia report no financial disclosures. Dr. Blanco has received reports support from Pfizer and GlaxoSmithKline. Dr. Artieda-Urrutia has received lecture fees from Janssen.

\section{Funding}

This study was funded in part by the National Alliance for Research on Schizophrenia and Affective Disorders (NARSAD), Fondo de Investigación Sanitaria (FIS)[grant number PI060092]; Fondo de Investigación Sanitaria FIS [grant number RD06/0011/0016]; ETES [grant number PI07/90207]; the Conchita Rabago Foundation, the Harriet and Esteban Vicente Foundation, the Spanish Ministry of Health, Instituto de Salud Carlos III, CIBERSAM (Intramural Project, P91B; Rio Hortega CM08/00170 -Dr. Hilario Blasco-Fontecilla), the Alicia Koplowitz foundation, and the $\mathrm{Na}$ tional Institutes of Health, USA [grant number K24MH072712]. 


\section{References}

1. Chaturvedi SK. Coprophagia in a schizophrenic patient: Case report. Psychopathology. 1988; 21(1): 31-3.

2. Beck DA, Frohberg NR. Coprophagia in an elderly man: A case report and review of the literature. Int J Psychiatry Med. 2005; 35(4): 417-27.

3. Weschler D. Manual for the Weschler Adult Intelligence Scale, Revised. New York: Psychological Corporation; 1981.

4. Sheehan DV, Lecrubier Y, Sheehan KH, Amorim P, Janavs J, Weiller E, et al. The Mini-International Neuropsychiatric Interview (M.I.N.I.): The development and validation of a structured diagnostic psychiatric interview for DSM-IV and ICD-10. J Clin Psychiatry. 1998; 59 Suppl 20: 22-33; quiz 34-57.

5. Bugle C, Rubin HB. Effects of a nutritional supplement on coprophagia: A study of three cases. Res Dev Disabil. 1993; 14(6): 445-56.

6. Ghaziuddin N, McDonald C. A clinical study of adult coprophagics. Br J Psychiatry. 1985; 147: 312-3.

7. Soave O, Brand CD. Coprophagy in animals: A review. Cornell Vet. 1991; 81(4): 357-64.
8. Zanarini MC, Frankenburg FR, Dubo ED, Sickel AE, Trikha A, Levin A, et al. Axis II comorbidity of borderline personality disorder. Compr Psychiatry. 1998; 39(5): 296-302.

9. Soeteman DI, Hakkaart-van Roijen L, Verheul R, Busschbach JJ. The economic burden of personality disorders in mental health care. J Clin Psychiatry. 2008; 69(2): 259-65.

10. Ogrodniczuk JS. New directions in treatment research for personality disorders: Effectiveness of different levels of care. Psychother Psychosom. 2011; 80(2): 65-9.

\footnotetext{
* Corresponding author:

Enrique Baca-García

Department of Psychiatry

IIS-Jimenez Diaz Foundation

Autónoma University Madrid

Avenida Reyes Católicos 2,

28040, Madrid, Spain

Tel: +349 15504800

Fax: +349 15504987

E-mail: ebacgar2@yahoo.es
} 